\title{
HIDDEN REASON OF PAINT SYSTEM DELAMINATION
}

\section{Agnieszka KRÓLIKOWSKA', Leszek KOMOROWSKI', Pier Luigi BONORA ${ }^{3}$,}

\author{
'IBDiM, Warsaw, Poland akrolikowska@ibdim.edu.pl \\ IIBDiM, Warsaw, Polandlkomorowski@ibdim.edu.pl \\ ${ }^{3}$ retired full professor, University of Trento, Italy bonorapierluigi@gmail.com
}

\begin{abstract}
In many instances paints interlayers delamination is attributed either to environmental or to application reasons. The composition as well as the structure, including morphology are always given for granted. Extended interlayer delamination was observed on two huge structures ( $>50000 \mathrm{~m} 2 \mathrm{each}$ ) causing more than \$10 M damages. Overcoating time, UV exposure, Solvent retention and other possible environmental possible causes were considered. However, a careful morphological analysis showed that substantial differences existed in terms of the resin to pigment ratio, the surfactants, the quality of pigments and fillers (dimensions, shape, wettability, reactivity, homogeneity of distribution...) with respect to the sample used to certify suitability of the systems to climatic conditions C4 - C5 (ISO12944). The need to avoid any either voluntary or casual modification in both PVC and the kind and size of pigments to be used should be made evident as well as shown in some paragraph of existing Standards.
\end{abstract}

Keywords: "delamination"; "CPVC"; "pigments"; "fillers"; "topography"

\section{Introduction}

Organic protecting coatings for structural steel are formulated and applied in billions of square meters to the aim of avoiding or at least delaying both aesthetic and structural degradation, providing a predictable long service life to bridges, plants, ships, cars ...Unfortunately, in many Countries paints are not classified as "building materials" and hence are not checked and certified by authoritative bodies, but their quality is instead left in the hands of "Standards" under the agreement and mutual control of the parties involved in a project. These Standards cover some specific properties and features and relate to "requirements" also agreed by the parties aimed to reach the expected durability in a classified aggressiveness of an environment.

The standards are mainly, if not exclusively, concerned with performances and related to such parameters as both number and thickness of layers, hardness, adhesion, gloss, scrub resistance, corrosion resistance, porosity etc.

Such parameters depend obviously on the care and wisdom in both formulation and proportions of components. This is not an easy task, from both scientific as well as technical and finally, economical point of view.

A typical protecting system is usually composed of a primer, an midcoat and a top coat.

Primers may be pigmented with metallic elements anodic to steel; primers are also used containing inhibitive pigments to interfere with the corrosion process.

Midcoats are specially designed to provide mechanical strength, to enhance the overall protection and, when highly pigmented, to decrease permeability to oxygen and water. The incorporation of laminar pigments, such as micaceous iron oxide, reduces or delays moisture penetration in humid atmospheres and improves tensile strength. Midcoats must remain compatible with finishing coats when there are unavoidable delays in applying them.

The quality of the protecting coating is built up by controlling the instable and complex equilibrium among the very many chemically reactive as well specifically useful components

A first qualitative approach to the virtuous symbiosis of liquid - solid components has been studied in the forties of last century in Germany and given an understandable and workable shape by Asbeck 
and Van Loo [1] who stated the importance for the good handling of Pigment Volume Concentration (PVC) in formulation:

\section{\% PVC = volume of mineral $\times \mathbf{1 0 0 ~ / ~ ( v o l u m e ~ o f ~ m i n e r a l ~ + ~ v o l u m e ~ o f ~ r e s i n ~ s o l i d s ) ~}$}

After their deep and careful approach to the problem, their conclusions are still valid and widely used. Many basic properties of paints are affected either positively or negatively by the PVC ratio, and namely by CPVC (Critical PVC) i.e. by the volume that the mineral, sum of pigments and extenders, occupies in the matrix and its ratio to the volume of binder.

At values lower than CPVC there is an excess of binder. This results in high gloss, good scrub resistance, good corrosion resistance, good blister resistance and good adhesion. At values over CPVC, there is insufficient binder to completely coat all the mineral particles. This results in low gloss, poor scrub resistance, poor corrosion resistance, poor blister resistance and poor adhesion. It is recommended that the coating be formulated below CPVC but not at CPVC, since properties change rapidly at CPVC, and slightest differences from batch to batch can result in major differences in properties [1].

A very interesting paper on the subject was written by Rodriguez and alii [2]. It will be important to report here their conclusions, based on EIS measurements: "Anticorrosion coating performance is affected greatly by PVC/CPVC ratio. It has been concluded that in high crosslinked systems it is recommended the use of low pigment content to obtain better anticorrosion properties although the final coating can be more expensive."

The importance of CPVC is outstanding, but unfortunately does not cover the full evidence of the problem In these papers $[3,4]$ it is pointed out that the effects of addition of minerals to paints and coatings depend on: mineralogy (chemistry, crystal structure, Mohs scale hardness, etc.) oil absorption, brightness, $\mathrm{pH}$ both value and variability, chemical inertness, refractive index, purity, soluble salts, particle size and particle size distribution; particle shape and aspect.

When such parameters are not taken into appropriate consideration, durability might be seriously affected, since the binding power of the resin might mask, in the short term, the lack of interface equilibrium and the defects are suitable to affect mainly adhesion in the medium - long period.

One should also consider that paint systems are tested and "certified suitable" according to ISO 12944 Standard on the basis of samples tested by an official laboratory, which will certify that the results "refer exclusively to the tested samples" complying to a given set parameters, including the physical-chemical-mechanical-morphological features of solids, a given PVC, leaving alone the quality of the binder as well of other additives.

In case of failures occurring in field, they are usually attributed to either environmental or application diversities, applying a "bona fide" concept to the possibility that a mistake in the pigment percentage, or a replacement of, say, good, wettable, plate, flaky talc with some cheap carbonate, a fine grain size with coarse, inhomogeneous fragments might have negatively affected both value and durability of the paint. A check of paint morphology might solve many doubts, as the following examples are aimed to show.

\section{Experimental}

In this paper, we'd like to point out, based on practical, on-site examples that the study of paint morphology on a microscale might be crucial for understanding the actual mechanisms of a failure like an early interlayer delamination. Often this phenomenon is attributed to either excessive time elapsed before overcoating, or to amine outcrop, or even to UV degradation and chalking or bad 
surface preparation. When all and each one of such events are likely to have happened, it appears not necessary to investigate on other possible mechanisms.

We will report about two events of extended failures (interlayer delamination):

In the first case, concerning partly immersed - partly exposed to marine atmosphere structures the extension of delamination was so high, and the possible reasons of it were so many, including UV, time of overcoating, possible polyamine outcropping, that no investigation was supposed necessary on possible defects in the formulation of the system. The Laboratory collected several paint samples recovered after detachment from various locations of the structure. A comparison was possible of such samples with other ones obtained using paints of the same label either present in the Laboratory as reference (certified HD IM according to ISO12944 Standard) or bought directly on the shop.

A second case concerned a structure inserted in a polluted urban environment where extended delamination occurred between an epoxy intermediate and siloxane topcoat certified as ISO12944 class HD C5. Several detached samples were collected and examined. They were compared with samples of the same label taken from a bridge where no failures were observed after twelve years of exposure in a HD C5 environment according to ISO12944.

\section{The morphology of the mineral components}

For both series of samples and for the related "standard" or "reference" samples, SEM micrographs of both the cross sections and of the surfaces detached from the overcoats were obtained using a JEOL 6010 LV with EDX Scanning Electron Microscope. The photos were analysed with a surface analysing program and the size and amount of filler/extender/pigment particles were counted and compared.

The analysed samples showed in this article were:

a) A first series of samples taken from a partly immersed - partly exposed to marine atmosphere where extended delamination was observed. An example is shown in the first row of Fig.1. It was a polyamine cured high built epoxy mastic intermediate coat (Norsok certificate for HD IM) which delaminated from the epoxy mastic primer (samples called $1 \mathrm{U}$ in the following).

b) A second series of samples of the same brand with the same label but directly bought on the market (samples called 1 in the following). An example is shown in the second row of fig.1.

c) One series of samples of epoxy intermediate and siloxane topcoat certified as complying with ISO12944 class HD C5 taken from a bridge where no failures were observed (samples called 2) (third row of fig.1)

d) A series of paint samples the same brand with the same label taken from a structure where extended delamination between intermediate and topcoat were observed (samples called $\underline{\mathbf{2 U}}$ in the following) (fourth row of fig.1)

\section{2 - Results and discussion}

We analysed the above described four cases of systems which were applied on the assumption they were suitable for long service life (high durability, HD) in harsh environment (C5, IM according to ISO12944 Standard). The two systems were formulated and composed according to different design, but in principle expected to produce the same performances. 
In case "2" no failure was found after 12 years. Samples produced with the same batch of paint in the laboratory gave the results which are shown in both SEM micrograph (third row of figure 1) and in topographical analysis. The good performances are clearly due to a correct use of the right amount of well distributed, low dimension, low concentration both pigments and extenders. They are also well wetted by the resin, with no void and no crevice between pigment and binder.

The failures claimed in case $\underline{\mathbf{2}} \mathbf{U}$ are coherent with an excess of pigment size and concentration, a coarse distribution of their dimensions, an excess crowding at the interface. Pigments (extenders) are in many instances clearly not wetted by binder. A typical example of such behavior is displayed in the fourth row of figure 1
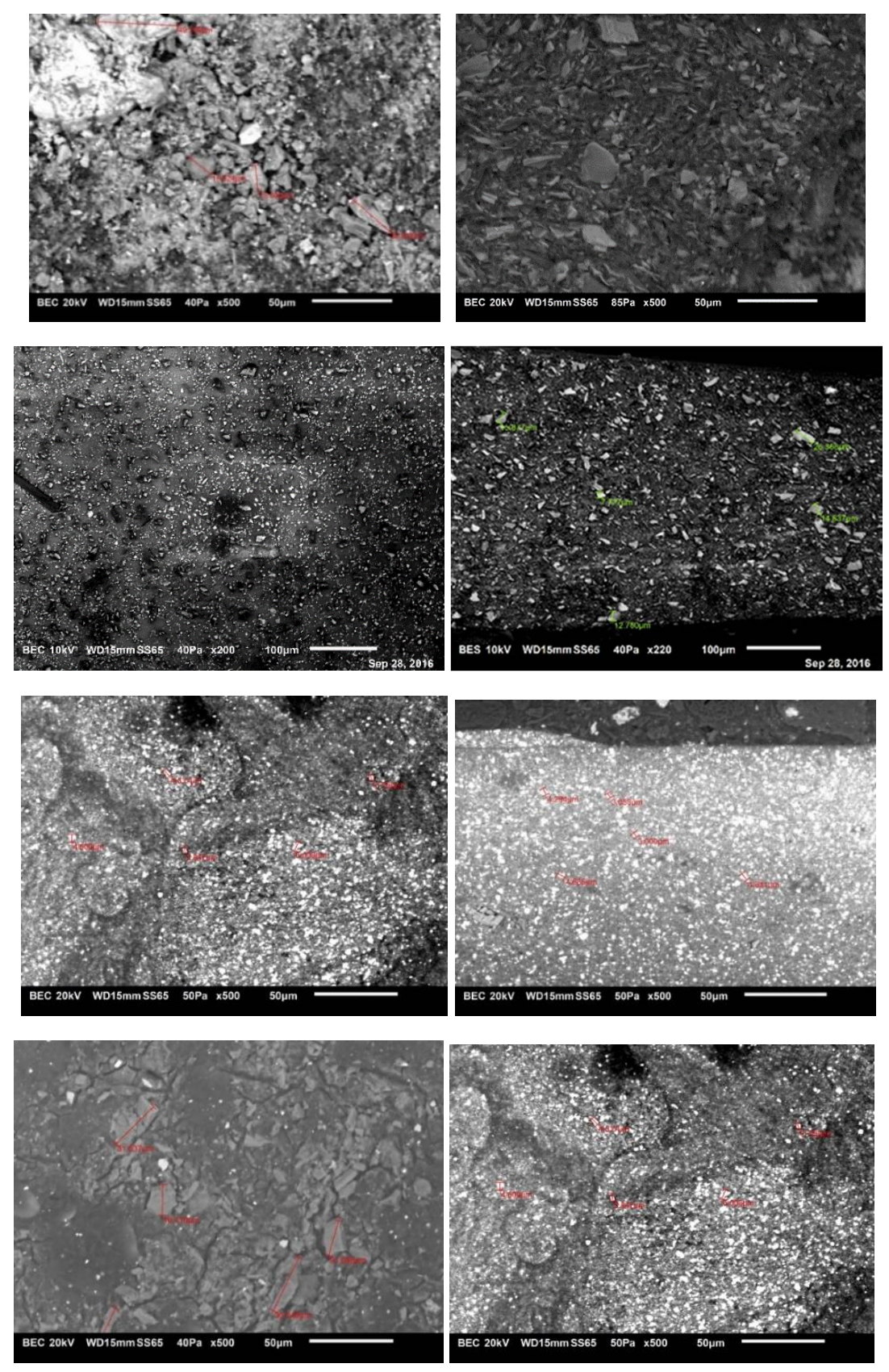

Figure 1 SEM micrographs of the surface (left) and of the cross section (right) of sample $1 \mathrm{U}$ (first row); sample 1 (second row); sample 2 (third row); sample $2 \mathrm{U}$ (fourth row) 
As for case $\underline{1 \mathrm{U}}$, a systematic morphological study of a reasonable number of samples collected in different locations where they delaminated (15 samples) provided evidence that a very low percentage of the resin in the interphase surface is left available for interlayer bonds by the coarse, crowded, partly wetted both extenders and pigments (detailed photo of pigment only partly wetted by binder is shown as Fig 2

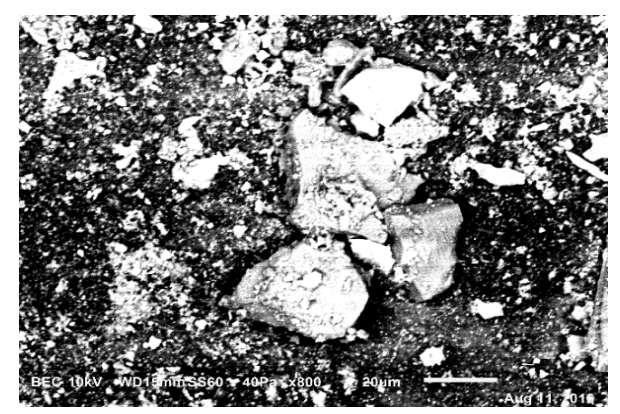

Fig.2: Fillers only partly wetted by the binder of sample $1 U$

\begin{tabular}{|c|c|c|c|c|c|c|c|}
\hline \multirow{2}{*}{ Sample } & \multicolumn{3}{|c|}{$\begin{array}{c}\text { Average } \\
\text { dimension of } \\
\text { the filler }[\mu \mathrm{m}]\end{array}$} & \multicolumn{2}{|c|}{$\begin{array}{l}\text { Number of the } \\
\text { fillers particles }\end{array}$} & \multicolumn{2}{|c|}{$\begin{array}{l}\text { The area taken } \\
\text { by fillers }\left[\mu^{2}\right]\end{array}$} \\
\hline & $\begin{array}{l}\text { On the } \\
\text { surface }\end{array}$ & \multicolumn{2}{|c|}{$\begin{array}{l}\text { On the } \\
\text { cross } \\
\text { section }\end{array}$} & $\begin{array}{l}\text { On the } \\
\text { surface }\end{array}$ & $\begin{array}{l}\text { On the } \\
\text { cross } \\
\text { section }\end{array}$ & $\begin{array}{l}\text { On the } \\
\text { surface }\end{array}$ & $\begin{array}{c}\text { On the } \\
\text { cross } \\
\text { section }\end{array}$ \\
\hline 1 & 9.92 & \multicolumn{2}{|c|}{20.77} & 1333 & 11286 & 94.85 & 279.82 \\
\hline $1 U$ & 47.30 & \multicolumn{2}{|c|}{74.66} & 892 & 1252 & 330.22 & 1410.06 \\
\hline 2 & 20.00 & \multicolumn{2}{|c|}{13.00} & 1640 & 11255 & 380.77 & 94.56 \\
\hline $2 U$ & 41.50 & \multicolumn{2}{|c|}{31.00} & 1409 & 4892 & 483.51 & 429.44 \\
\hline \multirow{2}{*}{ Sample } & \multicolumn{4}{|c|}{$\begin{array}{l}\text { Minimum diameter of } \\
\text { particles }[\mu \mathrm{m}]\end{array}$} & \multicolumn{3}{|c|}{$\begin{array}{l}\text { Maximum diameter of } \\
\text { particles }[\mu \mathrm{m}]\end{array}$} \\
\hline & \multicolumn{2}{|c|}{$\begin{array}{l}\text { On the } \\
\text { surface }\end{array}$} & \multicolumn{2}{|c|}{$\begin{array}{c}\text { On the cross } \\
\text { section }\end{array}$} & $\begin{array}{l}\text { On the } \\
\text { surface }\end{array}$ & \multicolumn{2}{|c|}{$\begin{array}{c}\text { On the cross } \\
\text { section }\end{array}$} \\
\hline 1 & \multicolumn{2}{|l|}{1.98} & \multicolumn{2}{|r|}{5.75} & 16.67 & \multicolumn{2}{|c|}{39.66} \\
\hline $1 U$ & \multicolumn{2}{|l|}{2.95} & \multicolumn{2}{|c|}{23.84} & 54.58 & \multicolumn{2}{|c|}{169.29} \\
\hline 2 & \multicolumn{2}{|l|}{8.17} & \multicolumn{2}{|c|}{7.52} & 55.76 & \multicolumn{2}{|c|}{34.62} \\
\hline $2 U$ & \multicolumn{2}{|l|}{16.20} & \multicolumn{2}{|r|}{9.79} & 101.17 & \multicolumn{2}{|c|}{90.60} \\
\hline
\end{tabular}

Table 1 - Topographical analysis of SEM micrographs

The comparison of the statistical topographical analysis (Table 1) gives an even more quantitative evaluation of the phenomenon. The SEM analysis with a suitable program of acknowledgement of different tonalities of grey allowed to evaluate the average dimensions of the particles, their number counted on both surface and cross section, the amount of area occupied by the particles and their both maximum and minimum diameter values.

A comparison of the values relevant to the defective and to the reference systems shows the tremendous existing differences. It looks like that the amount of space granted to the binder as well as the excess of roughness provided by the inerts to the interface, leaving alone the crevices left by the insufficient wetting (see e.g. fig.2) hindered the establishment of a durable interlayer bond. As an example, comparing the topographical results as related to samples 1 and $1 U$, it is evident that in the latter the dimensions of filler particles were such as to occupy an excessive percentage of the available area, even if their number was lower than in sample 1. Moreover, comparing for the same purpose samples 2 and $2 U$, it is evident that the dimensions of particles is also the responsible of 
early delamination, while in the case of samples $1 \mathrm{U}$, even worse effects were caused by an excessive concentration of coarse particle at the interface.

\section{3 - Conclusion}

Inert pigments, the ones which are neither anodic nor inhibitive, are not so a "sine cura", as it had been supposed until now. Their particle size, the presence of aggregates and agglomerates, their wettability by the binder and, consequently, their tendency to accumulate water at the pigment/binder interface if the film is subjected to water are important both to the durability and to the efficiency of a protecting coating.

The choice of the complements to resin in the formulation of a protecting coating is as important as the quality of the often much more expensive resin itself. It is therefore compulsory that PVC be far lower than CPVC, but also that minerals be of such sizes and dimensions as to avoid that their accumulation might reduce the space allowed to the binder necessary to assure a suitable and longlasting interlayer bond. The good paints performances are clearly due to a correct use of the right amount of well distributed, low dimension, low concentration of both pigments and extenders, which must be well wetted by the resin, with no void and no crevice between pigment and binder. The hard work of formulators and the careful strict control of certifying Laboratories might be made ineffective by the lack of check of the constancy of both composition and morphology between the certified sample and the applied batches of paint. The examples shown in this paper led to about 10 million Euro of damages. The need to avoid any either voluntary or casual modification in both PVC and the kind and size of pigments to be used should be made evident as well as shown in some paragraph of existing Standards.

\section{References}

1) W. K. Asbeck, M. Van Loo. Critical pigment volume relationship - Ind. Eng. Chem., 41 (7), (1949) 1470

2) M.T. Rodriguez, J.J. Gracenea, J.J. Saura - The influence of the critical pigment volume concentration (CPVC) on the properties of an epoxy coating: Part II. Anticorrosion and economic properties PROG. ORG. COAT., 50 (1) 2004, 68

3) Vanderbilt Minerals, LLC An Introduction to Mineral Fillers for Paints \& Coatings, PP: www.vanderbiltminerals.com/ee_content/Documents/Technical/PPT_Mineral_Fillers_Paints_C oatings_Web.pdf

4) F. McGonigle, P.A. Ciullo: INDUSTRIAL MINERALS AND THEIR USES" 1996 by Noyes Publications, chapt. 4, PAINTS \& COATINGS 